\title{
REALIZATION OF INTELLIGENT CONTROL FOR ROLL SHAPE IN ROLL GRINDER NC MACHINE
}

\author{
Liwen Yan ${ }^{1,2}$, Tao Yu ${ }^{1}, Y i \operatorname{Pan}^{2}$ \\ ${ }^{1}$ CIMS \& Robot Center of Shanghai University, Shanghai University, China; Email: \\ ylw@cslg.edu.cn. ${ }^{2}$ Mechanical Engineering Department, Changshu Institute of Technology, \\ China.
}

Abstract: During the roll grinding process, it is difficult to attain a high rolling shapes and surface quantities for several factors such as the emery wheel abrasion. This paper puts forward a new Neural Networks intelligent error compensation algorithm to measure round error, which is based on the analysis of the causation of rolling shape. And it has an experiment to verify the algorithm further.

Key words: Round, Neural Networks, Intelligent Compensation, Roll Grinder

\section{INTRODUCTION}

Rolls are used as tools themselves. They flatten size, shape or impart a finish to other materials like Steel, Aluminum, Paper, etc. In recent years, because of the metallurgy, deckle, rubber, plastics and print to dye the quick development of the industry economy of etc, there appear various rolls with different shapes of rolls, such as CIR, LINE, SINE, FREE and CVC curve roll. To meet the requirement of the market, the higher request is put forward to ensure the surface quantity and roll form accuracies of the different form for roll. For example, while whetting to pare the work roll, the target value with actual value deviation should be less than $\pm 1.0 \mu \mathrm{m}$, and while whetting to pare to prop up the roll, the target value with actual value deviation should be less than $\pm 1.5 \mu \mathrm{ms}$. The roll cylinder degree should be less than $0.005 \mathrm{~mm}$, and the radius direction deviation (roundness tolerance) should be less than

Please use the following format when citing this chapter:

Yan, Liwen, Yu, Tao, Pan, Yi, 2006, in International Federation for Information

Processing (IFIP), Volume 207, Knowledge Enterprise: Intelligent Strategies In Product Design, Manufacturing, and Management, eds. K. Wang, Kovacs G., Wozny M., Fang M., (Boston: Springer), pp. 505-510. 
$0.005 \mathrm{~mm}$, disallowing to grinding trace or chafe, etc, in the roll circumference direction.

Compared to other machine manufacturing methods, there are more and complex factors that affect the precision of manufacturing result for the complicated characteristics of grinding. In addition to the static state accuracy of the tool machine, there still has the e wear away of emery wheel, grinding force, the flexibility transform of craft system and some other factors, etc. In practical manufacturing process, while grinding, the influence of these factors is to be reflected in the roll form accuracy and the size error of the dimension. The actual result which we got indicates that characteristics as follows:

(1) The size of the roll has the trend of the gradual aggrandizement.

(2) The variety of the size of roll is not linear, even is not monotonous augmentative.

(3) On the same grinding machine, the size and the form varying regulations of the roll processed is also incompletely in phase.

From above, we can see that the higher request has been demanded to the numerical control roll grinder to meet the requirement of high-quality roll grinding. To make the roll grinding to be more accurate, more efficient, more automatic and more intelligent and to meet the requirements of the society and customers, we had developed a numerical control system of the roll grinder to accomplish the intelligent compensate control based on neural networks(NNs). The approaches of intelligent roundness error compensation of grinding roll realized as follow.

\subsection{The former feedback compensation control system of NC roll grinder}

In order to compensate the roundness error in the grinding, the methods used applied currently are showed in figure 1. Because of the production of roundness error of roll is nonlinear, this kind of simple way of error compensate can't offset the accidental factor and decrease the effect of roundness variety of roll by nonlinear factor, so we focus on the intelligent compensation system of shapes error of roll (including roundness error) based on neural networks.

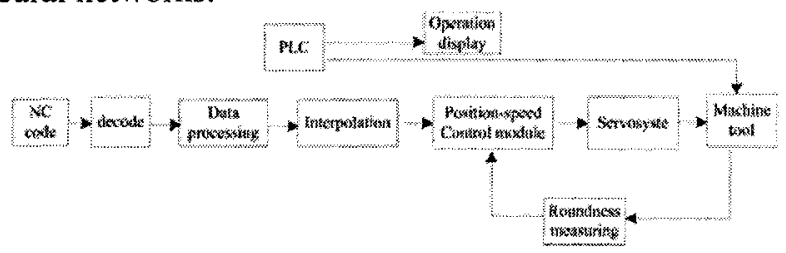

Fig. 1. Control system workflow of NC roll grinder 


\subsection{The adoption intelligence compensates system using NNs}

Artificial neural networks ( ANN ) comes into being the model from the microstructural and functional simulation of nervous system of human brain, which simulate the part capability of imagery thinking. Its features contain nonlinear characteristic, learning capability and self-adaptive, which is the most important way to simulate the intelligence of human being. For meeting the high performances of roll grinder, when the self-adaptive control is applied to real control process, where has many uncertain environment and manufacturing procedure, there are still some problem, for example, the complex structure of self-adaptive controller and the model reference adaptive control system (MRACS) can't insure zero steady-state error with certainty interference and etc.

In order to make full using of the self-adaptive control technology to improve the capability of robust, real-time, fault-tolerant control and the self-adaptive and learning ability for control parameters, and even more effectively control the process with many uncertainty and difficult to describe the nonlinear complex procedure, the self-adaptive control and NNs were combined together. Only the NNs directly act as controller in the control system can it utilize the advantage to construct perfect novel control system. ${ }^{[1]}$

\subsection{The working principle of the intelligent compensating system $^{[2][3][4]}$}

The neural network intelligent control system which we adopted is an error compensating system. As is shown in figure 2, the architecture of neural-network intelligent error-compensating system of roll roundness, $y_{d}, y$ are the standard roundness value and output roundness value of the roll in the system, $u$ is the control value. Normalized factor $K$ is added before and after the neural network, $(\mathrm{K}=\mathrm{kyd}, \mathrm{k}$ is the fine turning coefficient, generally span $0.5 \sim 1$ ), which made that the input value of the system is not limited, and made the system practical. The import values of the neural network are the normalized input value $y$ ' $d$ and the output values of three different time $y^{\prime}(k), y^{\prime}(k-1), y^{\prime}(k-2)$, which show the phase of the system.

The system remains the basic structure of neural network controller. The controller is a neural network, whose parameter, the connecting weight of neural network, can be adjusted online, and is not limited by the controlled object model structure, while the controlled object can be a non-linear, timevariant and unknown model. We can use the neural network as the 
compensating controller directly without a advance training, and the initial weight value get randomly from $[-0.1,+0.1]$. The system can adjust the weight value of the neural system, which made itself have a good adaptable capability.

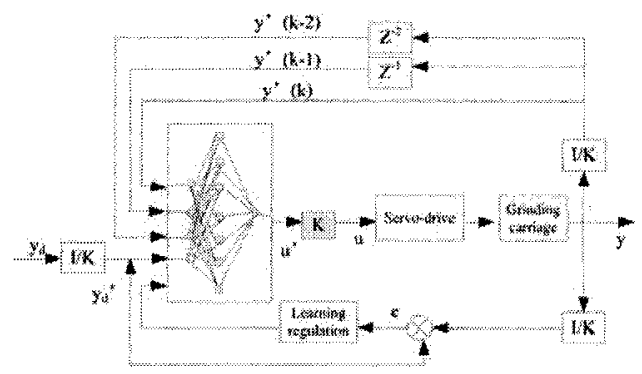

Fig. 2. Intelligent roundness error compensation based on NNs

\subsection{The roundness error compensating algorithm based on neural network}

\subsubsection{The formulation of nonlinear compensating control system}

The difference equations of nonlinear compensating control system are given as follows:

$$
y(k+1)=f\{y(k), y(k-1), \ldots, y(k-n+1)\}+\sum_{i=0}^{m} g_{i} \times u(k-i)
$$

Where $m \leqslant n ;$ The $\mathrm{g}_{\mathrm{i}}$ is constant coefficient; The outputs of system $y(k)$ $\in Y \subset R$; inputs of system is $u(k) \in U \subset R$, And have $f\{0\}=0$.

The output of NNs compensating controller is given by:

$$
\begin{aligned}
& u(k)=K\left(\sum_{j=1}^{N} V_{j} \times F\left(\sum_{j=1}^{N} P_{i j} \times X_{i}\right)-\theta\right) \\
& F(x)=1 /\left(1+e^{-x}\right) \\
& \theta=\frac{1}{2} \sum_{j=1}^{N} V_{j}
\end{aligned}
$$

Where $\mathrm{F}(\mathrm{x})$ is transfer function of hidden layers. $\theta$ is the threshold of output layer node which insure the stability of control system. 


\subsubsection{The learning algorithm of the NNs}

Define the quadratic function of error system e as value function $\mathrm{J}$ :

$$
\begin{aligned}
& J(K+1)=e(k+1)^{2} / 2 \\
& e(k+1)=\left(y(k+1)-y_{d}\right) / k
\end{aligned}
$$

The weight matrix of NNs is defined by:

$$
W=\left\{W_{i, j}\right\}=\left[\begin{array}{cccc}
V_{1} & P_{11} & L & P_{M I} \\
V_{2} & P_{I 2} & L & P_{M 2} \\
M & M & O & M \\
V_{N} & P_{I N} & L & P_{M N}
\end{array}\right]
$$

Regulate weights of NNs using the gradient descent algorithm, that is, adjust the descending direction of at the $J$ gradient:

$$
W_{i,}(k+1)=W_{i,}(k)-\beta \partial J(k+1) / \partial W_{i}
$$

Where $\beta$ is constant coefficient. Assumption:

$$
\begin{array}{r}
\Delta W_{i, j}(k+1)=-\beta \partial J(k+1) / \partial W_{i, j} \\
\Delta W_{i, j}(k+1)=-\beta \frac{\partial J(k+1)}{\partial u(k)} \cdot \frac{\partial u(k)}{\partial W_{i, j}}= \\
-\frac{\beta}{K} \cdot e(k+1) \cdot \frac{\partial y(k+1)}{\partial u(k)} \cdot \frac{\partial u(k)}{\partial W_{i, j}}=-\eta \cdot e(k+1) \cdot \frac{\partial u(k)}{\partial W_{i, j}}
\end{array}
$$

The learning algorithm of the nerve network can get from the type (2):

$$
\begin{aligned}
& \Delta V_{j}=-\eta \cdot e \cdot k\left(X_{j}-\frac{1}{2}\right) \\
& \Delta P_{i j}=-\eta \cdot e \cdot V_{j} \cdot\left(1-X_{j}\right) \cdot X_{j} \cdot X_{i}, i=1, \cdots, M, j=1, \cdots, N
\end{aligned}
$$

In order not to produce paralysis phenomenon of network, when NNs compensation controller works, the system error e is less than $\pm 5 \%$ and the control parameter $u$ overcome to the biggest threshold, the neural network would stop learning. 


\section{EXPERIMENTAL PARAMETERS AND ANALYSIS}

Machine for experiment, a roll grinder machine: MKZD84125

Specimen for grinding, length $1600 \mathrm{~mm}$, diameter $1000 \mathrm{~mm}$

The parameters of NNs compensation controller were set as:

The efficiency of study, $\mathrm{G}=0.1$

Nerve unit in the hidden layer, $\mathrm{N}=6$

Initialized value was selected randomly form $[-0.1,+0.1]$, and the coefficient of fine tuning $(\mathrm{k})$ was fixed at 0.65

Analysis on the experimental result:

The experiment on error compensation by NNs method was carried on the roll "SYZG02". When the intelligent error compensation was chosen, the outline of the roll fluctuated around the perfect outline and the stability of algorithm was nice. Since the error compensation just on roundness of the roll, there were several other parameters such as roundness error, size error, height error, roundness error and roughness error which should be considered in the real grinding process because the interrelationship exist among them. If the intelligent compensation by NNs was applied on all of the parameters that have been mentioned above, input more and output more could be achieved. The result from the experimental work showed the error margin shrink and the effectiveness on compensation was obvious.

\section{CONCLUSION}

The method of nerve network intelligent compensation could be applied on roll NC. After the nerve network was used, the ability of roll NC on handling the unknown nonlinear controlled device and self-adapting was improved. Such method could also be used in the control on other non-linear machines.

\section{REFERENCES}

1. Yang shuzi, Ding hong. Development and research of intelligence control technique and intelligences manufacturing system [J]. China Mechanical Engineering, 1992,3:(2) 1518.

2. Li shiyong. Fuzzy control - neural control and Intelligent Cybernetics [ M]. Harbin: Harbin Institute of Technology press, 1996. 169-178.

3. Zhang Ying, Zuo Li. Application of Neural Network in NC Machine Intelligent Control System [J]. Journal of Wuhan Transportation University, 1999, 23(3):226-229

4. Tian Xincheng $\mathrm{CNC}$ machining technique and control system of ceramic tools in: the Tongji University Proc of IEEE IC2IT'96 Shanghai: the Tongii University, 1996 34-36 